\title{
Clinical Outcomes of Arthroscopic Repair for Large or Massive Rotator Cuff Tear with Delamination: Comparison of Three Suture-Bridge Technique
}

Atsushi Okubo ( $\sim$ ma6-0336@nms.ac.jp)

Kyoto Kujo Hospital https://orcid.org/0000-0001-6994-5043

Tadahiko Yotsumoto

Kyoto Kujo Hospital

Nobuyoshi Watanabe

Nippon Medical School: Nihon Ika Daigaku

Teruyoshi Kajikawa

Kyoto Kujo Hospital

Shun Nakajima

Kyoto Kujo Hospital

Yasushi Oshima

Nippon Medical School: Nihon Ika Daigaku

Norishige lizawa

Nippon Medical School: Nihon Ika Daigaku

Tokifumi Majima

Nippon Medical School: Nihon Ika Daigaku

Research article

Keywords: rotator cuff tear, delamination, suture-bridge repair, en masse repair, dual-layer repair

Posted Date: February 24th, 2021

DOl: https://doi.org/10.21203/rs.3.rs-257609/v1

License: (c) (i) This work is licensed under a Creative Commons Attribution 4.0 International License.

Read Full License 


\section{Abstract}

Background: Rotator cuff tear with delamination is considered as a risk factor for postoperative retear after rotator cuff repair. The purpose of this study was to compare clinical outcomes between 3 repair procedures for large or massive rotator cuff tears with delamination: conventional en masse suture bridge (EMSB), double-layer suture bridge (DLSB), and combination of double-layer suture bridge and modified Debyere-Patte (DLSB+DP) methods.

Methods: A total of 53 shoulders of 52 patients who had large or massive rotator cuff tears with delamination were included. The patients were categorized into 3 groups: EMSB group comprised 18 patients, DLSB group comprised 24 shoulders of 23 patients, and DLSB+DP group comprised 11 patients. DP was applied for cases in which the rotator cuff was unable to be covered up to the footprint even though it was sufficiently mobilized. The mean postoperative follow-up period was 34.6 months (range, 24-72 months). Pre- and postoperative evaluations included the Constant scores and range of motion (ROM). Tendon integrity according to Sugaya classification, and fatty degeneration were also evaluated by magnetic resonance images (MRI).

Results: The ROM significantly improved after the operation in all groups. Mean constant scores significantly improved (from $45.5 \pm 14.3$ to $77.4 \pm 13.6$, in the EMSB, from $45.5 \pm 11.6$ to $87.6 \pm 11.4$ in the DLSB, and from $46.3 \pm 11.2$ to , and $88.0 \pm 10.5$ in the DLSB+DP). Significant differences were noted in the postoperative Constant score ( $p<0.05$ : DLSB vs. EMSB , and $p<0.05$ : DLSB+DP vs. EMSB) $₫$ The Constant pain score was better in the DLSB+DP than in the EMSB group. The mean pre-operative global fatty degeneration index was 1.52 in the EMSB group, 1.80 in the DLSB group, and 2.28 in the DLSB+DP group. Retear occurred in $27.8 \%$ in the EMSB group, $12.5 \%$ in the DLSB group, and $9.1 \%$ in the DLSB+DP group.

Conclusions: Comparison of 3 groups demonstrated that DLSB and DLSB+DP achieved better clinical outcome than EMSB. DLSB+DP is useful for large or massive rotator cuff tears with severe fatty degeneration or for cases where presence of excessive tension is anticipated when repairing the torn cuff.

\section{Introduction}

The delamination of the rotator cuff has been described as a horizontal lesion between the superficial and deep layers [1, 2]. Delamination is observed in 36-82\% of rotator cuff tear cases [3-9] and its presence is considered a risk factor for postoperative retear after rotator cuff repair [4]. Double-row double layer fixation was reported as a repair method of the stump of delaminated rotator cuff tears, but a high incidence of retear in large and massive rotator cuff tear cases has been known [2,10], and optimal fixation method for rotator cuff stump with delamination has yet been under debate. Suture bridge (SB) is a tendon stump fixation method for rotator cuff tears with a strong initial fixing force. It provides favorable cuff integrity by widely covering the footprint, being a widely performed general surgical procedure $[11,12]$. En masse suture bridge (EMSB) has been applied for the delaminated rotator cuff tear, and an experimental study has demonstrated the successful histological and biomechanical 
characteristics of cuff healing by this method [13]. We treated delamination of the rotator cuff with using EMSB method from 2010. However, it is unclear whether EMSB is appropriate for treating delaminated rotator cuff tears. Mochizuki, et al. stated the double layer suture bridge (DLSB), as it independently repairs the superficial layer (infraspinatus) and deep layer (articular capsule), which are anatomically different $[11,14]$. Some cases with EMSB method showed poor prognosis. To improve clinical results, we shifted the surgical method gradually from EMSB to DLSB.

The tear size and fatty degeneration of the rotator cuff are considered risk factors for retear after arthroscopic rotator cuff repair (ARCR) [15], and several repair methods of large or massive tears, such as musculotendinous advancement, have been reported [12, 16-19]. The modified Debeyre-Patte (DP) procedure was also reported effective for large or massive tears of the rotator cuff to reduce the tension of the repaired rotator cuff, which was applied for tears unable to be covered to the footprint by torn stumps even though sufficiently mobilized [19].

In the present study, we retrospectively evaluated the outcomes and cuff integrity of arthroscopic repair of delaminated rotator cuff tears employing the EMSB, DLSB, or DLSB combined with DP (DLSB + DP) in patients with large or massive delaminated rotator cuff tears. To our knowledge, there has been no comparative study including muscular advancement-combined DLSB for patients with degenerative delaminated rotator cuff tears. Our hypothesis was that DLSB would achieve a clinical outcome equal to or better than EMSB, and DLSB + DP would be optimal to reduce incidence of retear after repairing massive rotator cuff tears with delamination.

\section{Materials And Methods}

Among patients surgically treated by ARCR at our hospital, between September 2011 and June 2018, there were 53 shoulders (52 patients) presenting large or massive rotator cuff tears according to the DeOrio and Cofield classification with delamination. Inclusion criteria were large or massive rotator cuff tears undergoing ARCR and a minimum follow-up of 2 years. Exclusion criteria were patients underwent revision rotator cuff repair, and clinical findings of instability. Then, the subjects were classified into three groups for the treatment methods. The superficial and deep layers were fixed by en bloc suturing using suture anchors in the EMSB group, the superficial and deep layers were individually fixed by sutures similar to the method reported by Mochizuki et al. in the DLSB group [11, 14]. When the rotator cuff was unable to be covered up to the footprint even though it was sufficiently mobilized, the modified DP procedure [19] was added for fixation of the stump after DLSB following the method reported by Morihara et al., being designated as the DLSB + DP group. The EMSB group comprised 18 shoulders of 18 patients (12 males and 6 females at mean age of 69 years (range: 58-78 years)), the DLSB group comprised 24 shoulders of 23 patients ( 11 males and 12 females at mean age of 69.6 years (range: $49-87$ years)), and the DLSB + DP group comprised 11 shoulders of 11 patients (4 males and 7 females at mean age of 72.9 years (range: $67-87$ years)). The mean postoperative follow-up observation duration was 31.5 months (range: 24-67 months) in the EMSB group, 34.9 months (range: 24-72 months) in the DLSB group, and 26.7 months (range: $24-36$ months) in the DLSB + DP group (Table 1). 
The study was approved by an institutional review board, and the enrolled patients provided informed consent for participation in the study.

\section{Surgical Procedure:}

ARCR was performed in the beach chair position under general anesthesia in all patients. For retention of the upper limb, an arm holder (TRIMANO Arm Holder, Arthrex, Naples, FL, USA) was used. Five portals were mainly used for the operation (posterior, anterior, anterolateral, posterolateral, and upper anchor portals). For cases of subacromial decompression, subacromial synovectomy and subacromial osteophyte resection were mainly performed. Prior to repair, the rotator cuff was sufficiently mobilized. For the rotator cuff repair method, 1-2 medial anchors and 2-3 lateral anchors were set corresponding to the tear size using suture anchors (HEALIX, DePuy Synethes Mitek Sports Medicine, Raynham, MA, USA). In EMSB, the superficial and deep layers were fixed en bloc by passing the medial anchor thread. In DLSB, first, the medial anchor thread was passed through the deep layer to fix and suture by adding sliding knots and 2 half-hitches, as described by Mochizuki et al. [25], then all threads were passed through the superficial layer without cutting the anchor thread and fixed using the lateral anchor. In cases unable to be covered up to the footprint despite the rotator cuff being sufficiently mobilized, an approximately $3-\mathrm{cm}$ transverse skin incision was made outward from the medial border of the spine of the scapula following the method reported by Morihara et al. [19]. The modified DP procedure was added dissecting the supraspinatus and infraspinatus muscles, while maintaining continuity to the fascia of the rhomboideus muscle. Then, suture fixation of the stump was performed by DLSB.

\section{Rehabilitation:}

In the EMSB or DLSB group, a postoperative shoulder abduction brace was attached for 3-4 weeks corresponding to the tear size. In the DLSB + DP group, the abduction brace was attached for 6 weeks after surgery. Passive ROM training was initiated on the day following surgery, and active ROM training was initiated after detaching the abduction brace. Resistance exercise was initiated 12 weeks after surgery.

\section{Clinical Outcomes:}

We assessed the pre- and postoperative ROM (flexion, abduction, external rotation, internal rotation), Constant score as clinical outcomes, and global fatty degeneration index (GFDI) representing the preoperative degree of fatty infiltration into the rotator cuff observed on MRI as the mean of that of the supraspinatus muscle, infraspinatus muscle, and subscapularis muscle following the Goutallier classification [20,21]. In addition, postoperative cuff integrity was evaluated using the Sugaya's classification, and types IV and $\mathrm{V}$ were regarded as retear [22]. 


\section{Statistical analysis:}

For statistical analysis, EZR (Ver. 1.50, Saitama Medical Center, Jichii Medical University, Saitama, Japan) was used. The pre- and postoperative ROM, and the constant scores in each group were analyzed using the Mann-Whitney U-test, the Kruskal-Wallis was used for pre- and postoperative comparisons among the 3 groups, and Fisher's test was used for comparison of the incidence of retear among the 3 groups, setting the significance level to below $5 \%$.

\section{Results}

Each surgical group showed significant postoperative active ROM improvement, including flexion, external rotation, and internal rotation degrees (Table 2). In the EMSB group, mean range of motion before operation was flexion (Flex) $105.6^{\circ}$, external rotation (ER) $49.7^{\circ}$, internal rotation (IR) L 4.8; subsequent to the last follow-up, it was Flex $158.9^{\circ}$, ER $67.2^{\circ}$, IR L 2, respectively. In the DLSB group, ROM improved from Flex $109^{\circ}$, ER $47.2^{\circ}$, IR L 4.5 to Flex $159.6^{\circ}$, ER $71.4^{\circ}$, and IR L2, respectively. In the DLSB + DP group, ROM improved from Flex $108.2^{\circ}$, ER43.2 ${ }^{\circ}$, IR L3.6 to Flex149.1 ${ }^{\circ}$, ER 69. $1^{\circ}$, and IR L1.3, respectively. No significant difference was noted in both preoperative and postoperative ROM improvement among the 3 groups (Table 2).

The mean Constant scores were $45.5 \pm 14.3,45.5 \pm 11.6$, and $46.3 \pm 11.2$ in the EMSB, DLSB, and DLSB + DP groups before surgery, and $77.4 \pm 13.6,87.6 \pm 11.4$, and $88.0 \pm 10.5$ after surgery, respectively, achieving favorable improvement in all groups (Fig. 1A). Significant differences were noted in the postoperative Constant scores between the EMSB and DLSB groups $(p=0.018)$, and between the EMSB and DLSB + DP groups $(p=0.045)$. In addition, a significant difference was noted in the postoperative pain of the Constant score between the EMSB and DLSB + DP groups $(p=0.012)$ (Fig. 1B). No significant difference was noted in the postoperative ADL and Strength of the constant scores among the 3 groups (Figs. 1C and 1D).

The mean preoperative GFDI was $1.52 \pm 0.4,1.80 \pm 0.5$, and $2.28 \pm 0.4$ in the EMSB, DLSB, and DLSB + DP groups, respectively. GFDI in the DLSB + DP group was significantly higher than that of both EMSB and DLSB groups indicating significant preoperative fatty degeneration in the DLSB + DP group $(p=0.015$ : DLSB vs. DLSB + DP, and < 0.001: DLSB + DP vs. EMSB) (Table 3).

Postoperative retear occurred in 5 (27.8\%), 4 (16.0\%), and 1 patient $(9.09 \%)$ in the EMSB, DLSB, and DLSB + DP groups, respectively, showing the low incidence of retear in DLSB + DP group, while no statistical significant difference among the groups was found (Table 3). No other postoperative complication was observed.

\section{Discussion}

Rotator cuff tears with delamination are problematic for the risk of retear due to the anatomical characteristic of the horizontal superficial and deep layers. Many surgical procedures have been used 
while the optimal surgical procedure has not been determined. In this study, 3 surgical procedures, EMSB, DLSB, and DLSB + DP, were compared and investigated for their clinical outcomes. The retear rate was the lowest in DLSB + DP group, although the mean preoperative GFDI was the highest.

Suture bridge method has been reported to provide excellent rigidity compared with the single-row or double-row methods, because the contact area and contact pressure are greater [2, 23-27]; therefore, the suture bridge method has been frequently employed as a fixation technique for rotator cuff tear. However, the incidence of retear has been reported to be still high in cases of massive rotator cuff tear and severe fatty degeneration of the rotator cuff [15], and it remains controversial though several treatment methods were reported for irreparable rotator cuff tear by primary repair, such as partial repair [28], patch [12], musculotendon transfer [16], muscular advancement [19], and superior capsular reconstruction [18].

Delamination is often observed and tearing occurs in the posterior region in many cases [29]. For the developmental mechanism of the 2-layered structure, histologically, no consistent agreement has been obtained $[5,25]$. The rotator cuff has a structure comprising 5 histologically different layers, and delamination tends to develop between the 2nd and 3rd layers with different fiber distributions [30, 31]. Delamination is one of the risk factors for postoperative retear after rotator cuff repair and healing; thus, repair with special attention to the possible presence and state of delamination is considered important [3-5]. For the delamination repair, methods suturing the superficial and deep layers en bloc [32, 33], individually suturing the superficial and deep layers, and suturing only superficial layer $[8,14,34,35]$ have been reported. Mochizuki et al. reported that the superficial and deep layers should be individually sutured because the footprints of the 2 layers are anatomically different $[11,14]$. Some studies have described the usefulness of DLSB, comparing with EMSB and the bursal layer-only repair suture bridge (BLSB) $[6,8$, $35,36]$. This is based on the merit of DLSB in repairing the superficial and deep layers to an anatomically appropriate footprint [35]. Cha et al. stated a risk of retear in cases with severe rotator cuff degeneration even though the dual layer method was used, suggesting the necessity of another suture method and additional treatment for cases of severe degeneration of the rotator cuff [34].

In the present study, DLSB was performed and combined with the modified DP method for cases considered irreparable by the primary treatment. No significant difference was noted in postoperative ROM among the EMSB, DLSB, and DLSB + DP groups, but the postoperative Constant scores in the EMSB group were significantly inferior to those in the DLSB and DLSB + DP groups. The outcome of pain of the Constant scores in the EMSB group, which had the highest incidence of retear, was significantly inferior to that in the DLSB + DP group, which had the lowest incidence of retear. Statistically, the incidence of retear was not significantly different between the DLSB + DP group and the other 2 groups. However, in the DLSB + DP group, the retear ratio was the lowest while the preoperative GFDI was the highest and significantly higher than that of DLSB, suggesting the efficacy of modified DP method to reduce tension of the repaired rotator cuff and possibility of DLSB + DP to prevent retear in cases with severe degeneration of the rotator cuff. 
One of the limitations of our study is that we conducted retrospective investigation. There was a small sample size and a lack of a priori sample size calculation. In addition, the timing for the surgical choice of EMSB and DLSB might have influenced the clinical outcomes. However, to our knowledge, no study has conducted comparison of those methods with the muscular advancement-combined DLSB method for patients with degenerative delaminated rotator cuff tears. To further verify our findings, a long-term outcome evaluation is considered necessary.

\section{Conclusion}

Rotator cuff tears with delamination were treated by the EMSB, DLSB, and DLSB + DP method. The postoperative clinical outcomes and cuff integrity were evaluated. The DLSB method would be a better procedure capable of anatomical repair of delaminated rotator cuff tear of the shoulder. The DLSB + DP method may be a useful surgical procedure for patients with a large-sized tear, severe fatty degeneration of the rotator cuff, and excessive tension is present when repairing the rotator cuff.

\section{Abbreviations}

SB

suture bridge

EMSB

en masse suture bridge

DLSB

double-layer suture bridge

DP

Debyere-Patte

DLSB + DP

combination of double-layer suture bridge and modified Debyere-Patte

ARCR

arthroscopic rotator cuff repair

ER

external rotation

$\mathrm{IR}$

internal rotation

\section{Declarations}

- Ethical approval: The institutional review board of Kujo Hospital, Japan, approved to conduct this study.

- Consent for publication: Not applicable 
- Availability of data and materials: The datasets used and/or analysed during the current study are available from the corresponding author on reasonable request.

- Competing interests: The authors declare that they have no conflicts of interest regarding the present study.

- Funding: The authors received no special funding related to this study.

- Authors' contributions: Atsushi OKUBO designed the study, performed operations, acquired data, and analyzed results. Tadahiko YOTSUMOTO provided clinical advice and critically revised the contents of the manuscript. Nobuyoshi WATANABE, Teruyoshi KAJIKAWA, and Shun NAKAJIMA participated in the clinical treatment of patients and the improvement of this manuscript. Yasushi OSHIMA and Norishige IIZAWA made critical comments on the contents of the manuscript and assisted for completion of this manuscript. Tokifumi MAJIMA comprehensively reviewed and gave suggestions to improve this manuscript.

- Acknowledgements: Not applicable

- Informed consent: All subjects were explained and signed a written consent form.

\section{References}

1. Minagawa $\mathrm{H}$, Itoi E, Konno N, Kido T, Sano A, Urayama M, et al. Humeral attachment of the supraspinatus and infraspinatus tendons: An anatomic study. Arthroscopy. 1998.

2. Sugaya H, Maeda K, Matsuki K, Moriishi J. Functional and structural outcome after arthroscopic fullthickness rotator cuff repair: Single-row versus dual-row fixation. Arthrosc - J Arthrosc Relat Surg Arthroscopy. 2005;21:1307-16.

3. Boileau P, Andreani O, Schramm M, Baba M, Barret H, Chelli M. The Effect of Tendon Delamination on Rotator Cuff Healing. Am J Sports Med. SAGE Publications Inc.; 2019;47:1074-81.

4. Boileau P, Brassart N, Watkinson DJ, Carles M, Hatzidakis AM, Krishnan SG. Arthroscopic repair of full-thickness tears of the supraspinatus: Does the tendon really heal? J Bone Jt Surg - Ser A. J Bone Joint Surg Am. 2005;87:1229-40.

5. Han Y, Shin JH, Seok CW, Lee CH, Kim SH. Is posterior delamination in arthroscopic rotator cuff repair hidden to the posterior viewing portal? Arthrosc - J Arthrosc Relat Surg Arthroscopy. 2013;29:1740-7.

6. Heuberer PR, Pauzenberger L, Gruber MS, Ostermann RC, Hexel M, Laky B, et al. Delaminated Rotator Cuff Tears Showed Lower Short-term Retear Rates After Arthroscopic Double-Layer Repair Versus Bursal Layer-Only Repair: A Randomized Controlled Trial. Am J Sports Med. SAGE Publications Inc.; 2020;48:689-96.

7. Iwashita S, Hashiguchi H, Okubo A, Yoneda M, Takai S. Characteristics of the patients with delaminated rotator cuff tear. Sicot-J. 2018;4:28.

8. Kim YS, Lee HJ, Jin HK, Kim SE, Lee JW. Conventional en Masse Repair Versus Separate DoubleLayer Double-Row Repair for the Treatment of Delaminated Rotator Cuff Tears. Am J Sports Med. 
SAGE Publications Inc.; 2015;44:1146-52.

9. MacDougal GA, Todhunter CR. Delamination tearing of the rotator cuff: Prospective analysis of the influence of delamination tearing on the outcome of arthroscopically assisted mini open rotator cuff repair. J Shoulder Elb Surg J Shoulder Elbow Surg. 2010;19:1063-9.

10. Sugaya H, Maeda K, Matsuki K, Moriishi J. Repair integrity and functional outcome after arthroscopic double-row rotator cuff repair: A prospective outcome study. J Bone Jt Surg - Ser A. J Bone Joint Surg Am. 2007;89:953-60.

11. Nimura A, Kato A, Yamaguchi K, Mochizuki T, Okawa A, Sugaya H, et al. The superior capsule of the shoulder joint complements the insertion of the rotator cuff. J Shoulder Elb Surg J Shoulder Elbow Surg. 2012;21:867-72.

12. Nobuhara K, Hata Y, Komai M. Surgical procedure and results of repair of massive tears of the rotator cuff. Clin Orthop Relat Res. 1994;54-9.

13. Cheon SJ, Kim JH, Gwak HC, Kim CW, Kim JK, Park JH. Comparison of histologic healing and biomechanical characteristics between repair techniques for a delaminated rotator cuff tear in rabbits. J Shoulder Elb Surg Mosby Inc. 2017;26:838-45.

14. Mochizuki T, Nimura A, Miyamoto T, Koga H, Akita K, Muneta T. Repair of Rotator Cuff Tear With Delamination: Independent Repairs of the Infraspinatus and Articular Capsule. Arthrosc Tech. Elsevier B.V.; 2016;5:e1129-34.

15. Kim KC, Shin HD, Lee WY. Repair integrity and functional outcomes after arthroscopic suture-bridge rotator cuff repair. J Bone Jt Surg - Ser A. J Bone Joint Surg Am; 2012;94.

16. Gerber C. Latissimus dorsi transfer for the treatment of irreparable tears of the rotator cuff. Clin. Orthop. Relat. Res. 1992. p. 152-60.

17. McLaughlin HL. Lesions of the musculotendinous cuff of the shoulder. The exposure and treatment of tears with retraction. 1944. Clin Orthop Relat Res. 1994;3-9.

18. Mihata T, McGarry MH, Pirolo JM, Kinoshita M, Lee TQ. Superior capsule reconstruction to restore superior stability in irreparable rotator cuff tears: A biomechanical cadaveric study. Am J Sports Med Am J Sports Med. 2012;40:2248-55.

19. Morihara T, Kida Y, Furukawa R, Sukenari T, Kabuto Y, Kurokawa M, et al. Therapeutic outcomes of muscular advancement by an arthroscopic-assisted modified Debeyre-Patte procedure for irreparable large and massive rotator cuff tears. J Orthop Sci Elsevier. 2018;23:495-503.

20. Goutallier D, Postel JM, Bernageau J, Lavau L, Voisin MC. Fatty muscle degeneration in cuff ruptures: Pre- and postoperative evaluation by CT scan. Clin Orthop Relat Res [Internet]. 1994 [cited 2020 Jun 1]. p. 78-83.

21. Goutallier D, Postel JM, Gleyze P, Leguilloux P, Van Driessche S. Influence of cuff muscle fatty degeneration on anatomic and functional outcomes after simple suture of full-thickness tears. $J$ Shoulder Elb Surg Mosby Inc. 2003;12:550-4.

22. Sonnabend DH, Yu Y, Howlett CR, Harper GD, Walsh WR. Laminated tears of the human rotator cuff: A histologic and immunochemical study. J Shoulder Elb Surg Mosby Inc. 2001;10:109-15. 
23. Chen Y, Li H, Qiao Y, Ge Y, Li Y, Hua Y, et al. Double-row rotator cuff repairs lead to more intensive pain during the early postoperative period but have a lower risk of residual pain than single-row repairs. Knee Surgery, Sport Traumatol Arthrosc [Internet]. Springer Verlag; 2019 [cited 2020 Jun 22];27:3180-7. Available from: https://link.springer.com/article/10.1007/s00167-019-05346-0.

24. Mazzocca AD, Millett PJ, Guanche CA, Santangelo SA, Arciero RA. Arthroscopic single-row versus double-row suture anchor rotator cuff repair. Am J Sports Med Am J Sports Med. 2005;33:1861-8.

25. Park MC, ElAttrache NS, Tibone JE, Ahmad CS, Jun BJ, Lee TQ. Part I. Footprint contact characteristics for a transosseous-equivalent rotator cuff repair technique compared with a doublerow repair technique. J Shoulder Elb Surg J Shoulder Elbow Surg. 2007;16:461-8.

26. Park MC, Tibone JE, ElAttrache NS, Ahmad CS, Jun BJ, Lee TQ. Part II. Biomechanical assessment for a footprint-restoring transosseous-equivalent rotator cuff repair technique compared with a double-row repair technique. J Shoulder Elb Surg Elsevier. 2007;16:469-76.

27. Wall LB, Keener JD, Brophy RH. Clinical Outcomes of Double-Row Versus Single-Row Rotator Cuff Repairs. Arthrosc. - J. Arthrosc. Relat. Surg. Arthroscopy; 2009. p. 1312-8.

28. Burkhart SS. Arthroscopic treatment of massive rotator cuff tears: Clinical results and biomechanical rationale. Clin Orthop Relat Res. 1991;45-56.

29. Sonnabend DH, Watson EM. Structural factors affecting the outcome of rotator cuff repair. J Shoulder Elb Surg Mosby Inc. 2002;11:212-8.

30. Clark JM, Harryman DT. Tendons, ligaments, and capsule of the rotator cuff. Gross and microscopic anatomy. J Bone Jt Surg - Ser A [Internet]. 1992 [cited 2020 Jun 1];74:713-25.

31. Keisuke M, Ryo M, Nobuyasu O, Surgery O. Histological Assessment of Delamination Observed in Rotator Cuff Tears. Katakansetsu. 2006;30:461-4.

32. Gwak HC, Kim CW, Kim JH, Choo HJ, Sagong SY, Shin J. Delaminated rotator cuff tear: Extension of delamination and cuff integrity after arthroscopic rotator cuff repair. J Shoulder Elb Surg Mosby Inc. 2015;24:719-26.

33. Kwon J, Lee YH, Kim SH, Ko JH, Park BK, Oh JH. Delamination Does Not Affect Outcomes After Arthroscopic Rotator Cuff Repair as Compared With Nondelaminated Rotator Cuff Tears: A Study of 1043 Consecutive Cases. Am J Sports Med. SAGE Publications Inc.; 2019;47:674-81.

34. Cha SW, Lee CK, Sugaya H, Kim T, Lee SC. Retraction pattern of delaminated rotator cuff tears: Duallayer rotator cuff repair. J Orthop Surg Res. BioMed Central Ltd.; 2016;11.

35. Nakamizo H, Horie R. Comparison of En Masse Versus Dual-Layer Suture Bridge Procedures for Delaminated Rotator Cuff Tears. Arthrosc - J Arthrosc Relat Surg. W.B. Saunders; 2018;34:3150-6.

36. Kim SJ, Choi YR, Lee HH, Chun YM. Surgical Results of Delaminated Rotator Cuff Repair Using Suture-Bridge Technique with All-Layers or Bursal Layer-Only Repair. Am J Sports Med. SAGE Publications Inc.; 2016;44:468-73.

\section{Tables}


Table 1. Patient demographics

\begin{tabular}{|lllll|}
\hline & $\begin{array}{l}\text { EMSB } \\
(\mathrm{n}=18)\end{array}$ & $\begin{array}{l}\text { DLSB } \\
(\mathrm{n}=24)\end{array}$ & $\begin{array}{l}\text { DLSB + DP } \\
(\mathrm{n}=11)\end{array}$ & p-values \\
\hline Sex, $\mathrm{n}$ (males/females) & $12 / 6$ & $11 / 12$ & $4 / 7$ & .267 \\
\hline Dominant/non-dominant side & $12 / 6$ & $19 / 5$ & $7 / 4$ & .568 \\
\hline Age (years) & $69.0 \pm 6.1$ & $69.6 \pm 9.5$ & $72.9 \pm 6.1$ & .483 \\
\hline Follow-up period (months) & $31.5 \pm 14.0$ & $34.9 \pm 11.7$ & $26.7 \pm 4.7$ & .061 \\
\hline
\end{tabular}

Notes: Data are presented as the means \pm SD.

Table 2. Active range of motion before and after treatment

\begin{tabular}{|c|c|c|c|c|}
\hline & EMSB & DLSB & DLSB+DP & p-values \\
\hline \multicolumn{5}{|l|}{ Flex $^{\circ}$} \\
\hline Pre-operation & $105.6 \pm 50.0$ & $109 \pm 30.1$ & $108.2 \pm 43.2$ & .979 \\
\hline Last follow-up & $158.9 \pm 20.5$ & $159.6 \pm 19.7$ & $149.1 \pm 26.4$ & .509 \\
\hline \multicolumn{5}{|l|}{$\mathrm{ER}^{\circ}$} \\
\hline Pre-operation & $49.7 \pm 16.8$ & $47.2 \pm 20.0$ & $43.2 \pm 25.6$ & .623 \\
\hline Last follow-up & $67.2 \pm 13.7$ & $71.4 \pm 14.9$ & $69.1 \pm 10.8$ & .4 \\
\hline \multicolumn{5}{|l|}{ IR } \\
\hline Pre-operation & L 4.8 & L 4.5 & L 3.6 & .27 \\
\hline Last follow-up & L 2 & L 2 & L 1.3 & .502 \\
\hline
\end{tabular}

Note: Data are presented as the means \pm SD.

Abbreviations: Flex ${ }^{\circ}$ : degree of flexion; $\mathrm{ER}^{\circ}$ : degree of external rotation;

IR: internal rotation; L:Lumbar spine;

Table 3. Radiological findings on MRI 


\begin{tabular}{lcccc}
\hline & $\begin{array}{c}\text { EMSB } \\
(\mathrm{n}=18)\end{array}$ & $\begin{array}{c}\text { DLSB } \\
(\mathrm{n}=24)\end{array}$ & $\begin{array}{c}\text { DLSB + DP } \\
(\mathrm{n}=11)\end{array}$ & p-values \\
\hline & $1.52 \pm 0.4$ & $1.80 \pm 0.5$ & $* 2.28 \pm 0.4$ & $\leq .001^{*}$ \\
Preoperative GFDI & $5(27.8 \%)$ & $3(12.5 \%)$ & $1(9.1 \%)$ & .395 \\
Postoperative re-tear, $\mathrm{n}(\%)$ & $(* \mathrm{p}<0.05)$
\end{tabular}

Abbreviation: GFDI: global fatty degeneration index

$*:$ p-values $=\underline{0.015^{* 1}(\text { DLSB vs. DLSB }+D P)}, 0.107$ (DLSB vs. EMSB), and $\leq 0.001 * 2(D L S B+D P$ vs. EMSB)

GFDI in the DLSB+DP group was significantly higher than that of both EMSB and DLSB groups. 